\title{
Potencial Energy Functions \& Properties of Some Hydride Molecules
}

\author{
Ratikant Thakur \& Jagdhar Mandal* \\ University Department of Physics Tilka Manjhi Bhagalpur University, Bhagalpur-812007,Bihar,India
}

\begin{abstract}
The values of dipole moment $(\mu)$, rotational constant $\left(\alpha_{e}\right)$,vibrational constant $\left(\omega_{e} x_{e}\right)$, binding energy $\left(D_{i}\right)$ and dissociation energy $\left(D_{e}\right)$ have been computed using the four forms of shortrange repulsive interaction(SRRI) models with an aim to establish the applicability and validity of potential energy functions. The calculations have been done for chemically active but simple hydride namely, $\mathrm{LiH}, \mathrm{NaH}, \mathrm{KH}, \mathrm{RbH}, \mathrm{CsH}, \mathrm{BeH}, \mathrm{BH}, \mathrm{MgH}, \mathrm{AlH} \& \mathrm{SiH}$.The computed values are in fair agreement with the experimental values available in the literature for the mentioned system. The close agreement between the observed an the calculated values simply revealed that Hellmann and AliHasan forms of interaction models appear to be more appropriate short range repulsive interaction models for the prediction of many other properties of the system. This study shows that these models play an important role in molecular physics as well as in the problems of astrophysics.
\end{abstract}

Keywords: - Anharmonicity constant, Binding energy, Dipole-dipole \& Dipole-quadrupole interaction constants, Dissociation energy \& Rotational constant.

\section{Introduction-}

Alkali hydride molecules are chemically very simple compounds but due to their reactive nature, a very few physical properties of these molecules could be measured. As such the experimental information about them is not available in the literature. Owing to the non availability of the observed data for other practical applications the theoretical estimates of the various properties of these hydrides will be useful. In this connection several effects have already been taken up by different workers. The nature of forces which bind the atom or ions in the diatomic molecules is of fundamental importance in the fields of physico-chemical interest. Rittner [1] for the first time proposed a polarizable ion model for alkali halide molecules through which the interionic forces could be understood. Later on, Brumer \& Karplus [2] modified the Rittner model known as truncated Rittner model or simply known as T-Rittner model given by,

$$
U(r)=-\frac{z^{2} e^{2}}{r}-\frac{\left(\alpha_{1}+\alpha_{2}\right)}{2 r^{4}}-\frac{C}{r^{6}}-\frac{D}{r^{8}}+U_{R}(r)
$$

The 1 st term is the electrostatic attraction between two point charges $Z(+e) \& Z(-e)$ separated by an interionic distance $r$, the $2^{\text {nd }}$ term is the polarization energy, the third \& fourth terms are van der Waals dipoledipole and dipole-quadrupole interaction. The last term is the short-range repulsive interactions. $\alpha_{1}$ and $\alpha_{2}$ are the electronic polarizabilities of cation and anion respectively.An extensive work [3-7] has been done on TRittner model for alkali halide molecules but study of hydride molecules have not been properly taken up by theoretical workers.

There are several repulsive interactions proposed by different theoretical workers from time to time to describe the structure \& properties of diatomic ionic molecules [8-9].The repulsive interactions in logarithmic function [10-12] are also available in the scientific literature but the results are far from satisfactory. As a result, the exact form of short-range repulsive interaction model is still to be asscertained.

In the present paper we are using the Born-Mayer, Hellmann Varshni Shukla \& Ali-Hasan form of short-range overlap forms which involve potential parameters. These forms for the overlap term be exploited to describe the various physical properties of hydride molecular substances. Born Mayer [14-15] and HM [16] of repulsive interaction are exponential in nature capable enough to produce various properties of alkali halide molecules approximately. V.S. presented an alternative approach for potential energy function of diatomic molecules by assuming a term to represent the electrostatic interaction and other to represent repulsion arising from the overlap of outermost electrons of constituent atoms and ions. This potential has further been modified by introducing the concept of effective charge parameter. Ali \& Hasan [17] has developed an empirical short range repulsive interaction model. Which produced many molecular properties in close agreement with expt. Value. In our calculation we have been inspired to examine the applicability and suitability of the short range repulsive models incorporating the polarizable term and dipole-dipole and dipole-quadrupole vdW energy term 
which have not been studied by the previous workers taking all the terms in consideration. This is, therefore, the comprehensive study for the molecular hydride systems.

\section{Method Of Calculation}

A generalized formula for short-range repulsive interaction can be expressed as

$$
U_{R}(r)=\frac{B}{r^{m}} \exp \left(-\frac{r}{\rho}\right)
$$

Which takes the form of Born-Mayer repulsive potential when $m=0$, Hellmann repulsive potential when $m=1$ and Varshni-Shukla potential when $m=2$. Ali-Hasan empirical repulsive potential is expressed as

$$
U_{R}(r)=\frac{s}{r^{m}} \exp \left(-b r^{n}\right)
$$

In this potential $m=2$ and $n=\frac{3}{2}$. In equation (2) and (3) B,S, $\rho$ and $b$ are repulsive potential parameters. The repulsive potential parameters are determined by applying the equilibrium criteria:

$$
\left(\frac{\mathrm{dU}(\mathrm{r})}{\mathrm{dr}}\right)_{\mathbf{r}=\mathbf{r}_{\mathbf{e}}}=\mathbf{0}
$$

Where $K_{e}$ is the molecular force constant, $\omega_{e}$ is the equilibrium vibrational frequency $\mathrm{C}$ is the speed of light in vacuum, $\mu_{A}$ is the reduced mass and $r_{e}$ is the interionic separation.

The expressions for potential parameter obtained are given by

$$
\begin{array}{r}
x=K_{e} r_{e}^{2}+\frac{2 e^{2}}{r_{e}}+\frac{42 C}{r_{e}^{6}}+\frac{72 D}{r_{e}^{8}} \ldots \ldots \ldots \ldots \ldots \ldots \\
\mathrm{b}=\frac{1}{6 r_{e}^{\frac{3}{2}}}\left[(2 x-7)+\left(4 x^{2}+4 \mathrm{x}-47\right)^{1 / 2}\right. \\
\& \quad \mathrm{P}=\frac{2 r_{e}^{2} \exp \left(b r_{e}^{\frac{3}{2}}\right)}{3 b r_{e}^{3 / 2}+4}\left\lfloor\frac{e^{2}}{r}+\frac{6 c}{r_{e}^{6}}+\frac{8 D}{r_{e}^{8}}\right] \ldots \ldots \ldots \ldots
\end{array}
$$

The vdw dipole-dipole constant $\mathrm{C}$ and dipole-quadrupole constant D taken from Slater Kirkwood variational method [18] are expressed as

$$
\begin{gathered}
\mathbf{C}=\frac{\mathbf{3} \boldsymbol{e h}}{\mathbf{4 \pi m ^ { 1 / 2 }}} \frac{\boldsymbol{\alpha}_{\mathbf{1}} \boldsymbol{\alpha}_{\mathbf{2}}}{\left(\frac{\boldsymbol{\alpha}_{\mathbf{1}}}{\boldsymbol{N}_{+}}\right)^{\mathbf{1 / 2}}+\left(\frac{\boldsymbol{\alpha}_{\mathbf{2}}}{\boldsymbol{N}_{-}}\right)^{\mathbf{1 / 2}}} \\
\boldsymbol{E} \mathrm{D}=\frac{\frac{27 h^{2} \alpha_{1} \alpha_{2} h}{32 \pi^{2} m}\left(\left(\frac{\alpha_{1}}{N+}\right)^{1 / 2}+\left(\frac{\alpha_{2}}{N-}\right)^{1 / 2}\right)^{2}}{\left(\frac{\alpha_{1}}{N+}\right)-\frac{20}{3}\left(\frac{\alpha_{1} \alpha_{2}}{N_{+} N_{-}}\right)^{1 / 2}+\left(\frac{\alpha_{2}}{N_{-}}\right)}
\end{gathered}
$$

Where $N_{+}$and $N_{-}$are the effective no. of electrons in the ions are defined as

$$
\begin{aligned}
& \boldsymbol{N}_{+}=\mathbf{N}+Z_{+} \\
& N_{-}=\mathbf{N}-Z_{-}
\end{aligned}
$$

Here $\mathrm{N}$ is the total number of electrons in the outer two shells. The e $q^{n}$ (9) \&(10) are valid for the ions of $S$ block and p-block elements of Periodic Table. In the case of ions of d-block elements of Periodic Table,

$$
\mathrm{N}=N_{1}+0.5 N_{2}
$$

Where $N_{1}$ and $N_{2}$ are the number of electrons in the $1^{\text {st }} \& 2^{\text {nd }}$ outer shells respectively.

The rotational vibration coupling constant $\left(\alpha_{e}\right)$ and anharmonicity constant $\left(\omega_{e} \boldsymbol{x}_{\boldsymbol{e}}\right)$ are calculated by the formula,

$$
\begin{aligned}
& \alpha_{e}=-\left(\frac{X_{3} r_{e}}{3}+1\right) \frac{6 B e^{2}}{\omega_{e}} \ldots \ldots \\
\& \quad & \omega_{e} x_{e}=\left(\frac{5}{3} X_{3}^{2}-X_{4}\right) \frac{h}{64 \pi^{2} c \mu}
\end{aligned}
$$

Here $x_{p}=\left(\frac{d^{p} U(r)}{d r^{p}}\right)_{r=r_{e}} /\left(\frac{d^{2} U(r)}{d r^{2}}\right)_{r=r_{e}}$

The binding energy per mole $\left(D_{i}\right)$ and dissociation energy $\left(D_{e}\right)$ is given by

$$
\begin{aligned}
& D_{i}=-\mathbf{N U}\left(\boldsymbol{r}_{\boldsymbol{e}}\right) \\
& \text { \& } D_{e}=D_{i}-\mathbf{I}+\mathbf{E}
\end{aligned}
$$

Where $\mathrm{N}$ is the Avogadro`s number, $r_{e}$ is the equilibrium internuclear distance, $\mathrm{I}$ is the ionization potential and $\mathrm{E}$ is the electron affinity. 


\section{Results and Discussion}

The experimental input data for Interionic equilibrium separation $\left(r_{e}\right)$, Force constant $\left(K_{e}\right)$, Vibrational frequency $\left(\omega_{e}\right)$ and Rotional constant $\left(B_{e}\right)$ have been taken from Huber \& Hertzberg [19]. The values of electronic polarizibilities $\left(\alpha_{1}, \alpha_{2}\right)$ have been taken from Tessman [20].These deta have been shown in Table No-

$$
1
$$

\begin{tabular}{|c|c|c|c|c|c|c|}
\hline \multicolumn{7}{|c|}{ Table-1 } \\
\hline $\begin{array}{l}\text { Molecules } \\
\mathrm{LiH}\end{array}$ & $\begin{array}{c}\alpha_{1} \\
0.034\end{array}$ & $\begin{array}{c}\alpha_{2} \\
1.86\end{array}$ & $\begin{array}{l}B_{e} \\
7.53\end{array}$ & $\begin{array}{c}\omega_{e} \\
1367.4\end{array}$ & $\begin{array}{r}K_{e} \\
1.298\end{array}$ & $\begin{array}{r}r_{e} \\
1.596\end{array}$ \\
\hline $\mathrm{NaH}$ & 0.190 & 1.86 & 4.905 & 1352.0 & 1.080 & 1.887 \\
\hline KH & 1.143 & 1.86 & 3.412 & 1176.0 & $\mathbf{0 . 8 0 5}$ & \\
\hline 2.243 & & & & & & \\
\hline $\begin{array}{l}R b H \\
2.367\end{array}$ & 1.805 & 1.86 & 3.022 & 944.40 & 0.469 & \\
\hline $\begin{array}{l}R b H \\
2.367\end{array}$ & 1.805 & 1.86 & 3.022 & 944.40 & 0.469 & \\
\hline $\begin{array}{l}\mathrm{CsH} \\
2.494\end{array}$ & 2.989 & 1.86 & 2.752 & 1415.1 & 0.460 & \\
\hline $\begin{array}{l}\mathrm{BeH} \\
1.343\end{array}$ & 0.008 & 1.86 & 10.32 & 2060.8 & 2.263 & \\
\hline $\begin{array}{l}\text { BH } \\
1.232\end{array}$ & 0.003 & 1.86 & 12.02 & 2366.9 & 3.03 & \\
\hline $\begin{array}{l}\text { MgH } \\
1.730\end{array}$ & 0.094 & 1.86 & 5.62 & 1497.0 & 1.275 & \\
\hline $\begin{array}{l}\text { AlH } \\
1.646\end{array}$ & 0.052 & 1.86 & 6.40 & 1682.6 & 1.62 & \\
\hline $\mathrm{SiH}$ & 0.0165 & 1.86 & 7.50 & 2041.8 & 2.39 & \\
\hline
\end{tabular}

1.520
The computed values of dipole moments, van der Waals constant $C, D \&$ vdW energies are produced in

Table-2

Table-2

\begin{tabular}{|c|c|c|c|c|c|}
\hline Molecules & dipole moment $(\mu)$ & C & D & $W_{d-d}$ & $W_{d-q}$ \\
\hline LiH & 4.09 & 1.477 & 1.949 & 0.089 & 0.0465 \\
\hline $\mathrm{NaH}$ & 6.302 & 8.110 & 13.59 & 0.179 & 0.085 \\
\hline KH & 7.901 & 43.76 & 49.261 & 0.344 & 0.0769 \\
\hline $\mathbf{R b H}$ & 8.296 & 69.34 & 77.950 & 0.394 & 0.0791 \\
\hline CsH & 8.072 & 108.0 & 121.016 & 0.448 & 0.0808 \\
\hline ВеH & 1.476 & 1.18 & 0.523 & 0.242 & 0.049 \\
\hline BH & 0.413 & 0.14 & 0.206 & 0.041 & 0.0478 \\
\hline MgH & 5.164 & 13.72 & 4.40 & 3.924 & 1.02 \\
\hline AlH & 4.513 & 2.368 & 3.127 & 0.119 & 7.126 \\
\hline $\mathrm{SiH}$ & 3.598 & 0.78 & 1.10 & 0.063 & 0.047 \\
\hline
\end{tabular}

Table-3 presents the computed values of potential parameters for all the short-range repulsive potencial Table-3 parameters.

\begin{tabular}{|c|c|c|c|c|c|c|c|c|}
\hline \multirow{3}{*}{$\begin{array}{l}\text { Molecules } \\
\text { A.H }\end{array}$} & \multirow[b]{2}{*}{ values of } & \multicolumn{4}{|c|}{ Values of parameter $b$} & \multicolumn{3}{|c|}{ Values of parameter $\mathbf{P}$} \\
\hline & & B.M & Hell & V.S & A.H & B.M & Hell & V. \\
\hline & $(x)$ & $\left(\times 10^{8}\right)$ & $\left(\times 10^{8}\right)$ & $\left(\times 10^{8}\right)$ & $\left(\times 10^{12}\right)$ & $\left(\times 10^{-8}\right)$ & $\left(\times 10^{-20}\right)$ & $(x$ \\
\hline $\left.10^{-28}\right)(\times 1($ & & & & & & & & \\
\hline $\begin{array}{l}\text { LiH } \\
39.18\end{array}$ & 4.36 & 2.733 & 1.954 & 1.150 & 0.706 & 0.0275 & 134.32 & 58.77 \\
\hline $\begin{array}{l}\mathrm{NaH} \\
61.42\end{array}$ & 4.77 & 2.53 & 1.880 & 1.22 & 0.670 & 0.0349 & 201.23 & 115.45 \\
\hline $\begin{array}{l}\text { KH } \\
104.72\end{array}$ & 5.28 & 2.354 & 1.810 & 1.270 & 0.630 & 0.0519 & 332.65 & 231.89 \\
\hline $\begin{array}{l}\text { RbH } \\
120.10\end{array}$ & 5.395 & 2.279 & 2.198 & 1.26 & 0.60 & 0.0519 & 883.41 & 282.49 \\
\hline
\end{tabular}


Potencial Energy Functions \& Properties Of Some Hydride Molecules

\begin{tabular}{|c|c|c|c|c|c|c|c|c|}
\hline $\begin{array}{l}\text { CsH } \\
141.44\end{array}$ & 5.53 & 2.217 & 1.74 & 1.25 & 0.58 & 0.0572 & 446.08 & 345.82 \\
\hline $\begin{array}{l}\text { ВеH } \\
37.39\end{array}$ & 4.596 & 3.420 & 2.504 & 0.803 & 1.035 & 0.0394 & 158.91 & 31.52 \\
\hline $\begin{array}{l}\text { BH } \\
23.83\end{array}$ & 4.580 & 3.717 & 2.719 & 0.847 & 0.788 & 0.045 & 132.71 & 27.369 \\
\hline $\begin{array}{l}\text { MgH } \\
476.14\end{array}$ & 6.670 & 3.850 & 3.180 & 2.520 & 1.390 & 0.805 & 2960.47 & 1672.82 \\
\hline $\begin{array}{l}\text { AlH } \\
89.93\end{array}$ & 5.37 & 3.260 & 2.540 & 1.804 & 1.04 & 0.0610 & 317.10 & 73.34 \\
\hline SiH & 5.750 & 3.796 & 3.01 & 2.39 & 1.365 & 0.0914 & 435.37 & 208.55 \\
\hline
\end{tabular}

Table 4 displays the computed values of binding energy of the molecules with polarization.

\section{Table- 4}

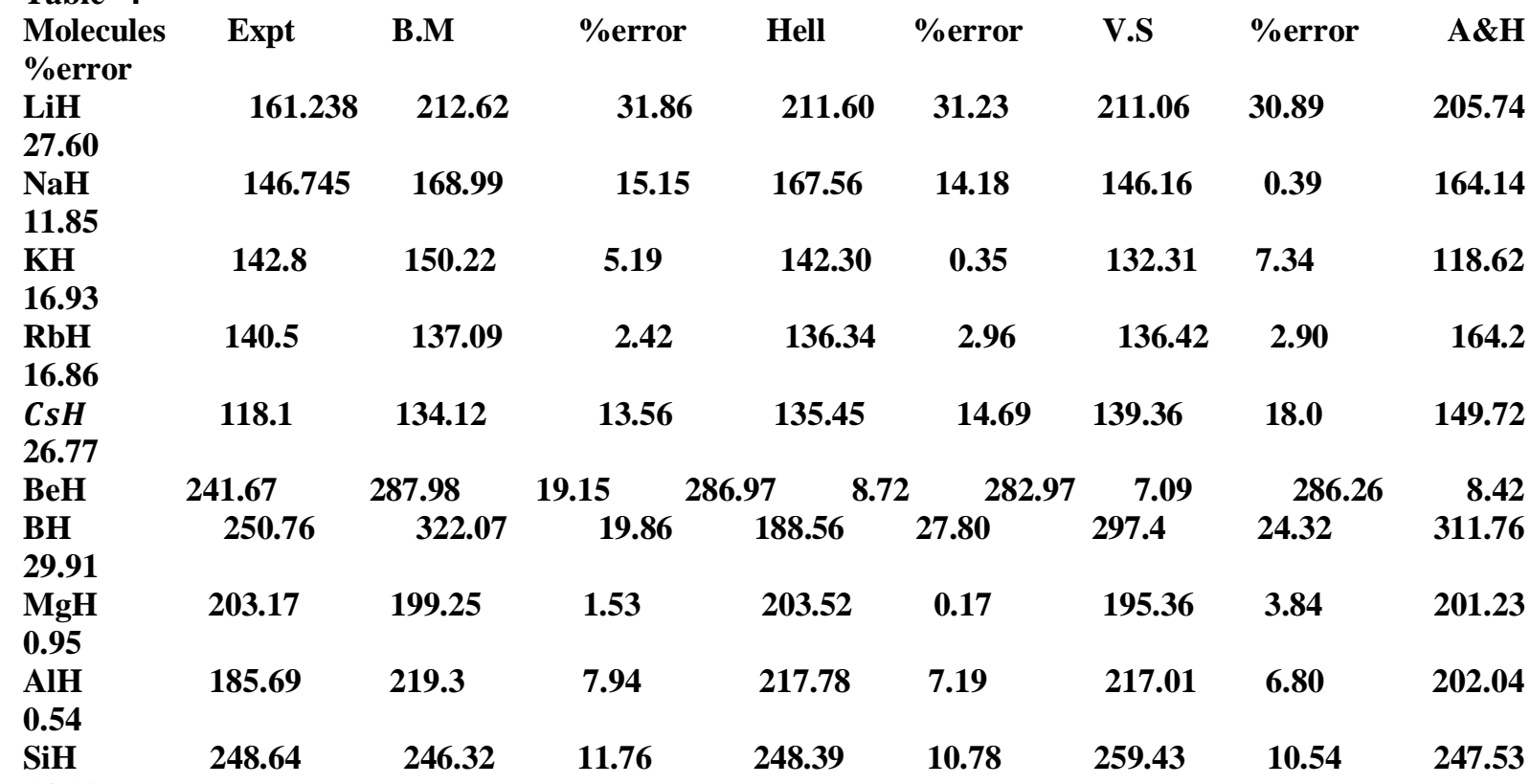

14.03

\begin{tabular}{|c|c|c|c|c|c|c|}
\hline & \multicolumn{6}{|c|}{ Table-5 presents the computed values of Dissociation energy of the molecules } \\
\hline Molecules & Expt. & B.M. & Hell & V.S. & & $\mathbf{A} \& \mathbf{H}$ \\
\hline LiH & 58 & 51.412 & 61.489 & 60.53 & & 103.916 \\
\hline $\mathbf{N a H}$ & 47 & 90.34 & 62.235 & 40.85 & & 58.84 \\
\hline KH & 42.9 & 86.72 & 70.47 & 63.48 & & 84.41 \\
\hline RbH & 39 & 75.24 & 74.47 & 74.10 & & 82.40 \\
\hline $\mathrm{CsH}$ & 41 & 60.54 & 42.44 & 86.4 & & 99.09 \\
\hline $\mathrm{BeH}$ & - & 330.60 & 326.4 & & 309.69 & 323.49 \\
\hline ВH & - & 61.45 & & & 466.59 & 526.92 \\
\hline MgH & - & 121.79 & 139.72 & 105.47 & & 130.09 \\
\hline AlH & - & 360.11 & 353.73 & 350.48 & & 287.65 \\
\hline $\mathrm{SiH}$ & - & 271.32 & 275.52 & 326.39 & & 276.43 \\
\hline
\end{tabular}

Table-6 shows the computed values of Rotational constants $\left(\alpha_{e}\right)$ in $10^{-4} \mathrm{~cm}$

\begin{tabular}{|c|c|c|c|c|c|c|c|c|}
\hline $\begin{array}{l}\text { Molecules. } \\
\text { A\&H }\end{array}$ & $\begin{array}{l}\text { Expt. } \\
\text { \% error }\end{array}$ & B.M. & \%eror & . & . & \%error & V.S. & \%error \\
\hline $\begin{array}{l}\mathrm{LiH} \\
17.1\end{array}$ & 0.2132 & 0.2144 & 0.67 & 0.245 & 4.78 & 0.218 & 3.76 & 0.259 \\
\hline $\begin{array}{l}\mathrm{NaH} \\
11.8\end{array}$ & 0.1353 & 0.138 & 2.27 & 0.128 & 5.18 & 0.147 & 8.1 & 0.162 \\
\hline KH & 0.0817 & 0.073 & 9.8 & 0.085 & 4.04 & 0.087 & 6.4 & 0.079 \\
\hline
\end{tabular}


Potencial Energy Functions \& Properties Of Some Hydride Molecules

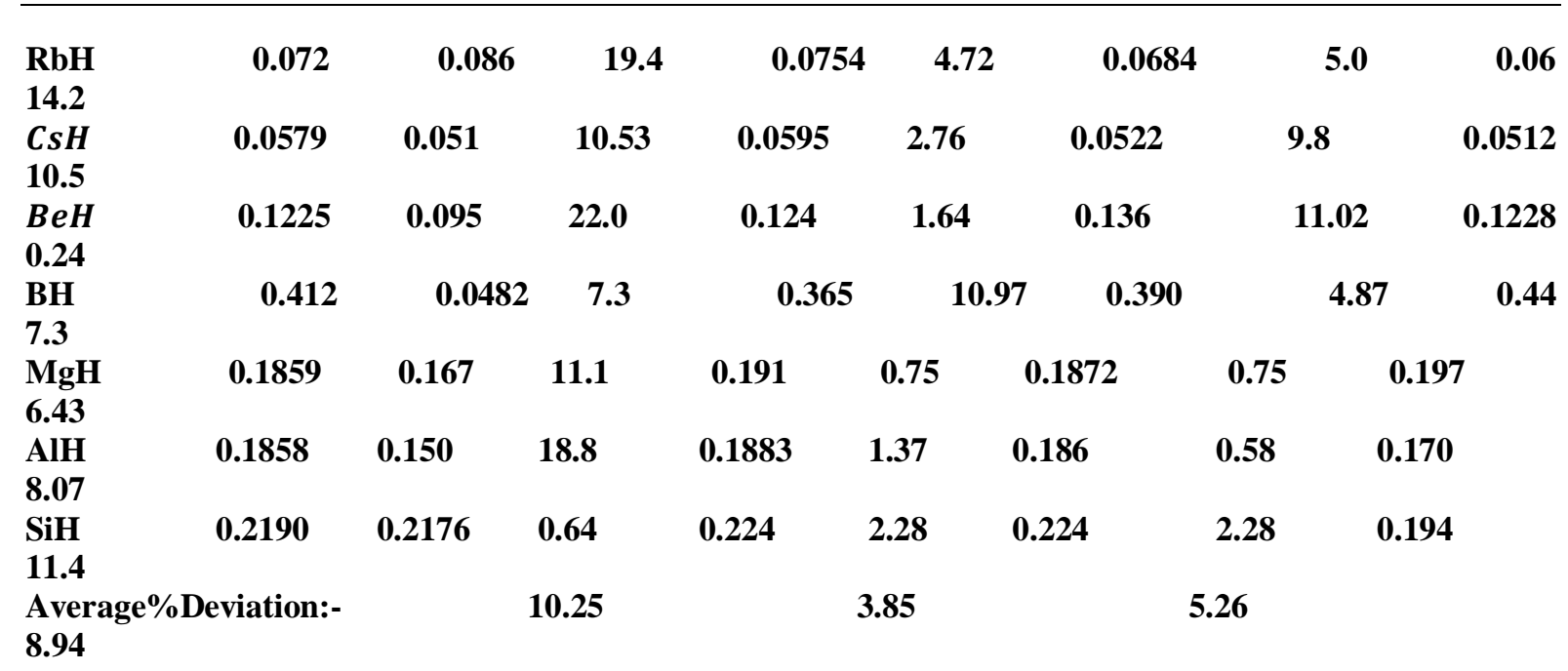

Table-7 computed values of vibrational Constant $\left(\omega_{\boldsymbol{e}} \boldsymbol{x}_{\boldsymbol{e}}\right)$ in $\mathrm{cm}^{-1}$

\begin{tabular}{|c|c|c|c|c|c|c|c|c|}
\hline \multirow{2}{*}{\multicolumn{2}{|c|}{$\begin{array}{l}\text { Molecules expt } \\
\text { A\&H \% error }\end{array}$}} & \multirow{3}{*}{$\begin{array}{l}\text { B.M. } \\
22.08\end{array}$} & \multirow{3}{*}{$\begin{array}{c}\% \text { error } \\
4\end{array}$} & \multirow{3}{*}{$\begin{array}{l}\text { Hell } \\
25.76\end{array}$} & \multirow{3}{*}{$\begin{array}{c}\text { \% error } \\
11.03\end{array}$} & \multirow{3}{*}{$\begin{array}{r}\text { V.S. } \\
27.72\end{array}$} & \multicolumn{2}{|c|}{$\%$ error } \\
\hline & & & & & & & & \\
\hline $\begin{array}{l}\mathrm{LiH} \\
21.03\end{array}$ & 23.2 & & & & & & 19.48 & 28.08 \\
\hline $\begin{array}{l}\mathrm{NaH} \\
12.98\end{array}$ & 19.72 & 17.41 & 11.71 & 15.08 & 21.05 & 16.19 & 17.90 & 17.16 \\
\hline $\begin{array}{l}\text { KH } \\
5.85\end{array}$ & 14.32 & 16.05 & 12.08 & 13.39 & 5.9 & 13.41 & 5.76 & 13.44 \\
\hline $\begin{array}{l}\text { RbH } \\
4.30\end{array}$ & 14.21 & 17.10 & 20.33 & 16.79 & 18.3 & 13.41 & 5.53 & 13.59 \\
\hline $\begin{array}{l}\text { CsH } \\
3.50\end{array}$ & 12.9 & 12.49 & 3.18 & 15.42 & 8.58 & 10.61 & 25.2 & 13.70 \\
\hline $\begin{array}{l}\text { BeH } \\
18.05\end{array}$ & 20.71 & 16.62 & 19.3 & 17.47 & 15.59 & 19.49 & 5.86 & 24.45 \\
\hline $\begin{array}{l}\text { BH } \\
14.09\end{array}$ & 49.39 & 53.01 & 7.32 & 51.03 & 3.31 & 45.05 & 8.78 & 42.43 \\
\hline $\begin{array}{l}\mathrm{MgH} \\
6.75\end{array}$ & 31.889 & 32.0 & 0.36 & 28.97 & 9.15 & 29.49 & 7.50 & 29.74 \\
\hline $\begin{array}{l}\text { AlH } \\
2.56\end{array}$ & 29.09 & 29.92 & 2.85 & 24.17 & 16.9 & 26.20 & 9.92 & 28.34 \\
\hline $\begin{array}{l}\mathrm{SiH} \\
3.83\end{array}$ & 35.51 & 39.18 & 10.3 & 44.49 & 25.3 & 38.27 & 7.78 & 36.86 \\
\hline Average & $\%$ deviation & & $9.14 \%$ & & $14.4 \%$ & & & $35 \%$ \\
\hline
\end{tabular}

B.M $>$ A.H $>$ V.S $>$ H.M

Table-6 \& 7 present the calculation of Rotational Constant $\left(\alpha_{e}\right) \&$ vibrational anharmonicity constant $\left(\omega_{e} x_{e}\right)$ respectively for all the four models taken into considerations. The percentage deviations are

also shown in the table along with the observed data. The experimental values of some hydride molecules are not available in the literature. There is good agreement between the experimental values and the calculated values of molecular properties of $\alpha_{e}, \omega_{e} x_{e}$ considering the approximations involved in the theoretical method and uncertainties associated in the experimental values of the parameter used. It is interesting to observe that most of the calculated values of $\alpha_{e}, \omega_{e} x_{e}$ are slightly more than the observed values. These may be due to the fact that the diatomic hydrides have less ionicity. The results have clearly improved by the inclusion of dipoledipole \& dipole- quadrupole interation energy terms [21]. These terms are essential for the interaction energy to be exact one.

\section{Acknowledgement.}

We are extremely thankful to Prof. Md. Mazahir Hasan to suggest the problems. 


\section{References :-}

[1]. Rittner E.S.J.chem. phys. 19(1951),1030

[2]. Brumer P.P. \& Karplus M, J.chem.phys.58(1973),3903

[3]. Shankar J, Kumar M \& Kaur A.J. Indian.J. Phys60B,1985,171

[4]. J.Shankar \& A.K.Rajaria, Indian journal Pure and Applied Physics, 20,1982,169-173

[5]. Gupta R.K, Kaur A.J, Bakshi p.s \& Shankar J. Indian J. Phys. Part B, 56, A82, 344

[6]. Kaur A.J, Jain P.C, Bakshi P.S \& Shankar J Indian J. chem.. Sec A, 22, 1983,595

[7]. Kaur A.J, Singh M, Bakshi P.S \& Shankar J. Indian J. Phys, Part B, 57, 1983,334

[8]. Varshni Y.P \& Shukla R.C. Rev.Mod. Phys.35,1963,130

[9]. Varshni Y.P \& Shukla R.C, J.Chem.Phys(USA),35,1961,582

[10]. Thakur K.P and Pandey J.P.J.Chem.Phys. 71, 1974,850

[11]. Thakur K.P \& Sarkar G.K. Indian Journal Pure and Applied Physics, 13, 1975,718

[12]. Thakur K.P.Aust J. Phys (Australia), 29, 1976, 39

[13]. Miss A.J. Kaur, P.C.Jain, P.S.Balchshi \& J.Shankar. Indian J. of Chemistry 22A, 1983, 595-598

[14]. Pandey R.P \& Pandey J.D Indian J.Chem. 20A, 1981, 591

[15]. Born Mayer \& Mayer J.E.Z.Phys. 75, 1932

[16]. Heiimann, Acta Chemica 2(1934), 91B J.Chem.Phys. 3, 1935, 61

[17]. Ali M.S \& Hasan M.M. Indian J.Phys. 63B, 1989, 486

[18]. Slater J.C. \& Kirkwood J.G. Phys. Rev-37,1931,682

[19]. Huber K.P. \& Hertzberg G, Constants of diatomic molecules (Van Nostrard-Reinhold co.Newyork, 1979)

[20]. Jack.R.Tessman \& A.H. Kahn, Physical Review, 92, 1953, 890-895

[21]. Raghuban shi S.S. \& Sharma L.K. Indian J. Pure \& Applied Phys, 16, 1978, 1071 